weise deshalb keine Kenntnis, weil die polizeilichen Datensammlungen, die ebenfalls zu Zeiten der Bekämpfung des (Links-)Terrorismus ausgebaut und mit enormen Datenmengen gefüllt wurden, nur wenig an Daten über die rechtsterroristische Szene enthalten. Das aber kann kein Grund dafür sein, Ermittlungen in dieser Richtung zu unterlassen. Daß sie viel zu spät erst einsetzen, zeigt das Beispiel der Überwachung der Republikaner durch den Verfassungsschutz: Nur zwei Bundesländer haben davon Gebrauch gemacht. Erst jetzt, wie vor wenigen Tagen den Nachrichten zu entnehmen war, soll das bundesweit ausgedehnt werden.

III. Mein Fazit lautet also: Wir haben aus strafrechtlicher Sicht keinen zusätzlichen Handlungsbedarf. Was wir brauchen, ist die Erinnerung daran, daß Polizei und Staatsanwaltschaft von Gesetzes wegen in Anwendung der geltenden Strafgesetze zu ihrer Verfolgung verpflichtet sind. Das Strafrecht hat danach kein sogenanntes »Ausländerproblem« sondern ein Inländerproblem: Die letztlich verfassungsrechtlich vorgegebene Pflicht zur unparteilichen Verfolgung von Straftaten, also zur Gleichbehandlung des Unrechts ist verletzt, wenn die Verfolgung davon abhängig gemacht wird, welchem Bereich des politischen Spektrums Gewalttaten zugeordnet werden.

Ursula Nelles

\title{
Überlegungen zum Rechtsradikalismus '92 aus sozialpsychologischer Sicht
}

In einer Phase globaler historischer Veränderungen sind die Deutschen, vor allem die Nachkriegsgenerationen, extrem verunsichert. Gründe dafür: die schon seit langem schwelende Strukturkrise, zunehmende Arbeitslosigkeit, Ausbildungs- und Wohnungsnotstand, allgemeine Entfremdungsprozesse, Europäische Einigung, Deutsche Vereinigung, Zerfall der Ostblockstaaten und resultierende Flüchtlingsströme, Afrika-Krise, Umweltzerstörung, AIDS, und und und. Der »Kalte Krieg « war zwar bedrohlich. Offenbar noch bedrohlicher ist es aber, keine klare, stabile Ordnung von Freund und Feind mehr zu haben. Es entsteht, nicht zuletzt auch durch manche unverhältnismäßige Dramatisierung in den Medien, zunehmend tiefe, subjektiv als existenzbedrohend erlebte, kollektive Angst.

Angst ist ein sehr unangenehmer Affekt. Sie muß angesichts der Ohnmacht, die Bedrohung unmittelbar zu beseitigen, innerpsychisch bewältigt, oder wie es psychoanalytisch heißt, abgewehrt werden. Zivilisierte, reife Formen der Angstbewältigung bestehen in besonnener Problemanalyse, vernünftigem gesellschaftlichen Diskurs und politischer Willensbildung hinsichtlich sinnvoller Lösungsmöglichkeiten. Je intensiver das subjektive Erleben von Bedrohung, desto stärker die Tendenz zum Zurückdrängen reifen seelischen Funktionierens, zur Regression, zu Vorurteilen, zu primitiven, irrationalen, panikartigen, gewalttätigen Reaktionsformen. Ob solche Regressionen entstehen, sich generalisieren, wie weit sie gehen - all das hängt zu einem gewissen Grade von der individuellen Persönlichkeit, sehr weitgehend aber auch von gesellschaftlichen und massenpsychologischen Einflüssen ab. Ein Hauptmerkmal solcher Abwehr ist jedenfalls das enorme unbewußte Potential der Menschen, etwas in andere Menschen oder in Situationen hineinzuphantasieren, die Tendenz zu Projektion und Abspaltung: Da wo die objektive Realität nicht unmittelbar und schnell beeinflußbar erscheint, wird sie eben ohne Aufschub psychisch umgestaltet. 
Wir haben es bei dem beobachtbaren Syndrom von Fremdenhaß, Antisemitismus,

Rechtsradikalismus und Neo-Nationalismus mit einem historischen und durchaus auch spezifisch deutschen Ineinandergreifen von fünf unterscheidbaren Grundmustern solcher Projektion und Abspaltung zu tun.

1. Die Bedrohungsgefühle werden abgewehrt durch kollektive symbiotische Verschmelzungsphantasien, durch Regression auf eine archaische Geborgenheitsphantasie, auf eine idealisierte, geschlossene, homogene familienartige Gemeinschaftlichkeit: auf die Nation. Angst- und Ohnmachtsgefühle werden abgewehrt durch in der Nation aufgehobene Allmachts- und Größen-Phantasien, durch projektiv-idealisierende Stilisierung von »starkem « Führer und "Vaterland «, an deren Strahlkraft man partizipieren kann.

Damit verwirklichen sich auch unbewußte narzißtische Vorstellungen: Nur wer mir blutsverwandt, »völkisch « gleich ist, ebenso rein und ideal, ist wertvoll; das kulturell oder individuell Andersartige bedroht diese Phantasien und muß entwertet und ausgegrenzt werden. Dazu gehören auch Reinheits-Ideologien vom "gesunden Volkskörper « ebenso wie das Phantasma von der "ethnischen Säuberung «. Diesen Mechanismus hat sich insbesondere der Faschismus zunutze gemacht.

2. Der Nationalsozialismus, sein menschenverachtender Rassismus und Massenmord von Völkern, Minderheiten, Kriegsfeinden etc. - all dies ist in den zwei bis drei Generationen der Nachkriegszeit psychisch kaum reflektiert und verarbeitet worden. Es ist stattdessen mehr oder weniger tabuisiert und kompensiert worden, vor allem mit Wohlstand. Insbesondere konnte nicht über das Entsetzliche getrauert werden, konnten die Kränkungen und Erniedrigungen der »schuldigen « Elterngeneration nicht verarbeitet werden. Es mußte alles abgewehrt werden, was daran gemahnte. Das förderte zum einen die untergründige Re-Mythisierung der $\gg \mathrm{Na}-$ tion «, die mit einer Art Erlösungshoffnung besetzt war und sich dank der Tabuisierung nicht an der Realität der multikulturellen Gesellschaft der BRD zu messen brauchte.

Zum anderen wurden deswegen insbesondere die Juden, aber auch andere Gruppen wie Intellektuelle, Linke und Linksradikale (Studentenbewegung) usw., die als Mahner und Warner an die Öffentlichkeit traten, als unbequeme, ja quälende Zeitgenossen erlebt. Soweit diese das kollektiv Verleugnete bewußt zu machen suchten, wurden sie abgewehrt, entwertet, verfolgt. So entstand unter der Hand auch eine Art sekundärer Anti-Semitismus unter der Oberfläche des offiziellen "Philo-Semitismus : der Haß auf diejenigen, die einem Schuldgefühle machen, und daraus folgend die kollektive Schuldumkehr, d. h. eine Projektion von Schuldgefühlen.

Die Wiedervereinigung scheint nun das Phantasma der Nation neuerlich Wirklichkeit werden zu lassen: In einer Art magischen Reinigungsrituals soll die Nation wiedererstehen, soll endlich all das Lästige vergessen sein, die Vergangenheit endgültig "entsorgt « sein. Die darin enthaltene Realitätsverleugnung ist umso stärker, je mehr die faktische Entwicklung der Einheit von den anfänglichen Suggestionen abweicht.

3. Mit diesem Grundmuster des Nationalismus - der Projektion idealisierter Selbstanteile und dem Reinheits-Mythos - eng verknüpft ist die uns allen eigene, allgegenwärtige, gleichsam allägliche Angstabwehr durch Projektion und Abspaltung negativer Selbstanteile: Die eigenen als schwach, böse, schmutzig, minderwertig usw. empfundenen Anteile werden etiketthaft projiziert auf dafür sich eignende Außengruppen wie Ausländer, Andersfarbige, Behinderte, Homosexuelle etc. Mit diesen konstruierten Außenfeinden, Prügelknaben, werden die verpönten Selbstanteile sodann »ausgemerzt«.

4. Die erwähnten Bedrohungen sind teils real: soziale Deklassierung usw., teils 
phantasiert. Jedenfalls bewirken sie primär miserable Gefühle von Ohnmacht, von Entwertung im ganzen Kollektiv. Die einfachste Lösung ist, die wahren Ursachen zu verschieben, den Fremden zum Verursacher des Übels und mithin zum Feind zu erklären: Die psychische Stabilisierung resultiert aus der Phantasie, mit der Vertreibung oder Vernichtung des Feindes, des Sündenbocks, seien auch die Probleme beseitigt.

5. Die fünfte Form der Projektion sehe ich in einer Art Delegations- oder Beauftragungsverhältnis. Was von den beschriebenen Grundmustern in großen Teilen der Bevölkerung noch latent bleibt, drückt sich doch mehr oder weniger implizit in den Medien oder in Äußerungen mancher Politiker aus. Seit Jahren ist in zunehmend entwertender Begrifflichkeit und aggressiver Tönung von Ausländern, ScheinAsylanten etc. die Rede. In Politiker-Reaktionen auf Rostock oder Mölln heißt es doppelzüngig: "Das schadet unserem Ansehen im Ausland!« oder »Mit türkischen Arbeiterinnen hat es nun wirklich die Falschen getroffen! « Dadurch entstanden Bahnungen für Über-Ich-Entlastung, für die Vorstellung, die Grundlogik der Ausmerzung könne nicht ganz falsch sein. Auch wenn es sich dabei nicht um bewußte "Beauftragung « handelt: Die Generation beziehungsloser Kinder, "vergessener « Jugendlicher, ungefestigter Adoleszenten ist real von Arbeits- und Sinnlosigkeit bedroht, verängstigt, aggressiv. Sie sind doch zugleich bedürftig nach Anerkennung und Identifizierung: Sie nehmen die »Botschaft« auf und setzen sie in Handlung um. Eine gelungene Projektion der Aggressivität der Elterngeneration.

\section{Was tun?}

Strafrecht als ausschließlich reaktives und repressives Instrument muß das Gegenteil dessen bewirken, was damit bezweckt ist, Strafrecht grenzt aus und spaltet ab. Es reproduziert und intensiviert damit auf gesellschaftlichem Niveau die geschilderten destruktiven Abwehr-Mechanismen.

Die eigentliche Be- und Verarbeitung von Sozialproblemen muß an deren Bedingungen ansetzen. Statt dies sachgerecht zu flankieren, wird das Strafrecht gern als "Mittel der Wahl « eingesetzt. Wenn es nicht funktioniert, gibt es "Mehr von demselben!«. Es wird nach noch mehr Strafrecht gerufen, genau wie es für Rückfällige höhere Strafen geben zu müssen scheint, anstatt die umgekehrte Schlußfolgerung zu ziehen: Wenn es nicht funktioniert, muß man vielleicht etwas anderes probieren als Strafrecht. Die ausschließliche Benutzung von Strafrecht verleitet dazu, die Sozialprobleme auf Individualprobleme zu verkürzen, das Strafrecht zum Stellvertreter für Sozialpolitik zu machen.

Wenn überhaupt Einsatz des Strafrechts etwas bewirken kann, dann gewissermaßen im unspezifischen Sinne, nicht als Strafrecht, sondern als überhaupt rechtsförmige, die Menschenrechte wahrende individuumsbezogene Intervention. Sozialen Sinn kann solcher Eingriff nur dann entfalten, wenn er einen Verarbeitungsprozeß in Gang setzt, nicht als abschließende Problemverarbeitung mißverstanden wird.

Insoweit kann eine klare rechtsstaatliche Reaktion allgemeine Zivilisations-Normen bekräftigen: Individual- und kollektivpsychologisch kann das zum angstmindernden und positive Identifikationen fördernden Erleben von »guter Autorität«, von Besonnenheit, Konsistenz und klarer Grenzsetzung beitragen. Das kann aber nur gelingen, wenn zugleich an den realen sozialen Bedingungen der Bedrohungsangst angesetzt wird und ein offener gesellschaftlicher Diskurs um Werte, Ziele und Mittel diese eigentlichen Gründe nicht mehr verleugnet. 